\title{
DOSSIER
}

De Prácticas y discursos Universidad Nacional del Nordeste Centro de Estudios Sociales I Año 8, Número 11, 2019, Marzo I ISSN 2250-6942

\section{DERIVAS CRIMINOLÓGICAS DE LA GUBERNAMENTALIDAD: MAPAS DEL DELITO Y GRILLA DE INTELIGIBILIDAD ECONOMICISTA'}

\section{CRIMINOLOGICAL DRIFTS OF GOVERNMENTALITY: CRIME MAPS AND GRID OF ECONOMIC INTELLIGIBILITY}

\section{Federico Luis Abiuso?}

RESUMEN Tomando como punto de partida las elaboraciones de Michel Foucault, en este artículo propongo describir dos de las derivas criminológicas de la gubernamentalidad. La primera de ellas vinculada a una de las tecnologías mediante la cual se pretende gobernar la cuestión criminal: la construcción de mapas del delito. La segunda, referida a la aplicación de una grilla de inteligibilidad economicista sobre los comportamientos delictivos y a la definición del delincuente como homo economicus. Ambas fueron reconstruidas a partir del análisis de dos registros documentales producidos desde Argentina, y con eje en el ámbito de la ciudad de Buenos Aires: el Segundo Informe de Conflictividad (2010), producido por el Ministerio Publico Fiscal de la Ciudad de Buenos Aires, y algunos artículos de la Revista de Policia y Criminalística, editada entre 1995 y 2011 por la propia editorial de la Policía Federal Argentina.

Palabras clave: gubernamentalidad, racionalidades políticas, tecnologías de gobierno.

\footnotetext{
' Artículo recibido el 30 de septiembre de 2018. Aprobado el 16 de abril de 2019 .
}

\footnotetext{
${ }^{2}$ Licenciado y Profesor en Sociología, doctorando en Ciencias Sociales (Universidad de Buenos Aires, Argentina). Becario de investigación UBACyT del Instituto de Investigaciones Gino Germani (1IGG). Correo electrónico: abiusofederico@yahoo.com.ar.
}

\section{INTRODUCCIÓN}

En un artículo titulado muy elocuentemente Gubernamentalidad, Nikolas Rose, Pat O'Malley y Mariana Valverde (2012) esbozan las características principales de la perspectiva de pensar el poder político en términos de gubernamentalidad. Tomando como punto de partida las nociones que Michel Foucault presentó al respecto en los cursos dictados en el Collège de France, Seguridad, territorio, población (ciclo lectivo 1977-1978) y Del gobierno de los vivos (1979-1980), los autores mencionan distintos ejemplos de este enfoque: análisis efectuados por Foucault y sus colaboradores -Françoise Ewald, Alessandro Fontana, Pasquale Pasquino, entre otros- en torno a la razón de estado, la ciencia de policia y el arte de gobierno liberal. Para ellos, pensar el poder político desde la perspectiva de la gubernamentalidad nos invita a descentralizar nuestra mirada respecto del Estado, apuntando más bien a una multiplicidad de autoridades que gobiernan en distintos sitios y en relación con diferentes objetivos. Así, lejos de considerar a esta perspectiva analítica como un corpus teórico cerrado e internamente homogéneo acerca del poder, la autoridad o el gobierno, Rose, O'Malley y Valverde (2012: 117) sostienen que "plantea preguntas particulares sobre los fenómenos que intenta comprender, preguntas susceptibles de ser respondidas a través de investigación empírica". Considero que un terreno fértil para plantearse distintas preguntas-problemas, ampliando de esta manera los horizontes y a la vez los desafíos del heterogéneo campo de los estudios en gubernamentalidad, es el de sus derivas criminológicas.

Apoyándome en una de las elaboraciones conceptuales presentadas por Foucault (2011a: 136), entiendo por gubernamentalidad:

El conjunto constituido por las instituciones, los procedimientos, análisis y reflexiones, los cálculos y las tácticas que permiten ejercer esa forma bien específica, aunque muy compleja, de poder que tiene por blanco principal la población, por forma mayor de saber la economía política y por instrumento técnico esencial los dispositivos de seguridad. 
De Prácticas y discursos

Universidad Nacional del Nordeste

Centro de Estudios Sociales

Año 8, Número 11, 2019, Marzo

ISSN 2250-6942

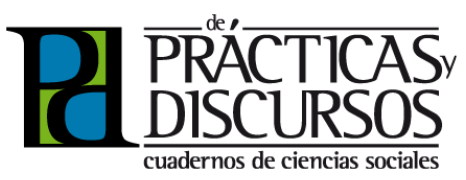

ABSTRACT Taking as a starting point the elaborations of Michel Foucault, in this article

I propose to describe two of the criminologi-

cal drifts of governmentality. The first of them

linked to one of the technologies through

which it is intended to govern the criminal

question: the construction of crime maps. The

second, referred to the application of a grid of

economic intelligibility on the criminal beha-

viors and the definition of the delinquent as

homo economicus. Both of them were recons-

tructed from the analysis of two documentary

records produced from Argentina, and with fo-

cus in the field of the City of Buenos Aires: Se-

gundo Informe de Conflictividad (2010), pro-

duced by the Fiscal Public Ministry of the City

of Buenos Aires, and some articles of Revista

de Policia y Criminalística, published between 1995 and 2011 by the own editorial of the Ar-

gentine Federal Police.

Keywords: governmentality, political rationa-

lities, governmental technologies.
Dicha forma de ejercicio de poder había sido presentada en anterioridad, en ocasión del curso dictado en el Collège de France, en el ciclo lectivo 1975-1976 (titulado Defender la sociedad), donde Foucault (2010) puntualizaba -en la última clase del mismo- la emergencia de una tecnología de poder distinta a la anatomopolítica, la biopolítica, cuyo objeto de intervención es el cuerpo múltiple de la población, y aquellos fenómenos colectivos a los cuales este estaría asociado (natalidad, fecundidad, morbilidad, enfermedad, vejez, etc.); el interés estará con respecto a estos fenómenos en las previsiones, las estimaciones estadisticas, las mediciones globales. Similares observaciones realizaba en el primer volumen de Historia de la sexualidad (La voluntad de saber, 1976), al destacar las dos formas principales (o los dos polos) a partir de las cuales se desarrolló, desde el siglo XVII, un biopoder o poder sobre la vida: las disciplinas del cuerpo y los controles o regulaciones de la población (Foucault, 2011b).

Esta acepción de gubernamentalidad, vinculada a la modalidad biopolítica de ejercicio del poder, es recuperada por Valverde (2017) en un reciente libro acerca de los aportes del pensamiento de Foucault al campo de saber criminológico. Al distinguirla de otras dos modalidades de poder-saber (soberanía y disciplina), por gubernamentalidad refiere a "modos de gobernar que son menos intrusivos y personales que la disciplina, y que se basan más en gestionar riesgos, organizar espacios para minimizar el desorden y las oportunidades delictivas, maximizar recursos y planificar para el futuro" (Valverde, 2017: 24, traducción propia). Dada la centralidad atribuida a los saberes, se mencionan a las estadísticas y los cálculos de probabilidad como algunas de las formas de saber característicos de dicho ejercicio del poder, aspectos que encontramos igualmente desarrollados por Foucault en los cursos Defender la sociedad y Seguridad, territorio, población.

Siguiendo lo expuesto por la autora, en ocasiones los vínculos entre las nociones de gubernamentalidad y biopolitica se vuelven más estrechos, al punto de que pueden solaparse entre sí. Especialmente en cuanto a su objeto de intervención respecta, puesto a que "ambos modos de gobernar dependen en gran medida de los datos agregados, y ambos modos son orientados al futuro" (Valverde, 2017: 90, traducción propia). Más aún, en estudios empíricos de proyectos gubernamentales concretos -sostiene Valverdeuno puede encontrar que no existe una frontera real que divide la biopolítica de la gubernamentalidad; tal distinción, meramente 
De Prácticas y discursos

Universidad Nacional del Nordeste

Centro de Estudios Sociales

Año 8, Número 11, 2019, Marzo

ISSN 2250-6942
Derivas criminológicas de la gubernamentalidad:

Mapas del delito y grilla de inteligibilidad economicista

Criminological drifts of governmentality:

Crime maps and grid of economic intelligibility

\begin{abstract}
${ }^{3}$ Mitchell Dean (2010) presenta un punto de vista similar al afirmar que, en la obra de Foucault, pueden diferenciarse dos sentidos de la noción de gubernamentalidad, uno históricamente delimitado y otro más general.

${ }^{4}$ Siguiendo lo expuesto por Senellart (2011), si bien hacia 1979 esta grilla todavía era aplicada en el marco del problema del Estado, en el curso del año siguiente -Del gobierno de los vivos, ciclo lectivo 1979-1980es extendida a una multiplicidad de formas de gobierno: de los niños, de las almas o las conciencias, de una casa, un Estado o de sí mismo. Un aspecto que iría marcando paulatinos desplazamientos en los temas abordados por Foucault, del "gobierno de los otros" hacia el "gobierno de sí mismo".
\end{abstract}

analítica, permite subrayar que la primera de estas modalidades de ejercicio del poder no tiene ningún apego particular a las ideas liberales de la ley o los mecanismos económicos de libre mercado (ilustrativamente, regimenes totalitarios pueden hacer uso de medidas biopolíticas en maneras que irian en contra de la lógica propia de la gubernamentalidad liberal).

Si bien estos últimos aspectos pueden dar lugar a mayores discusiones conceptuales, ellas exceden por lejos las pretensiones del presente artículo; por el momento, solamente querría recuperar aquella elaboración conceptual que vincula entre sí a la gubernamentalidad con la regulación de las poblaciones. Retomando al respecto tanto a Foucault, Valverde y a otro autor, Alessandro de Giorgi $(2005,2006)$ quien, igualmente proveniente del campo del saber criminológico -y con miras a indagar en las transformaciones acaecidas en las estrategias penales contemporáneas-, se apoya asimismo en la antedicha acepción, la cual fue citada de la clase del $1^{\circ}$ de febrero de 1978 de Seguridad, territorio, población. Esta elaboración no agota los términos a partir de los cuales Foucault presentó aquello que entendia por gubernamentalidad. Otras de las nociones son reseñadas justamente en la Situación de los Cursos que acompañan las ediciones de Seguridad, territorio, población y Nacimiento de la biopolítica. Al respecto, Michel Senellart (2011: 447) señala que "el concepto de 'gubernamentalidad' se desliza de manera gradual de un sentido preciso, históricamente determinado, a una significación más general y abstracta". En otras palabras, es desplazado desde un carácter acontecimental y regional, vinculada a capítulos especificos de la historia occidental -la introducción en el siglo XVIII de aquel régimen de poder que tenía por principal blanco la población, el proceso que llevó a que el tipo de poder de 'gobierno' predomine sobre todos los demás y el desarrollo de técnicas de gobierno que sirvieron de base a la formación del Estado moderno- hacia una definición en sentido amplio ${ }^{3}$.

De esa manera, en Nacimiento de la biopolítica, Foucault (2012: 218) afirma que la gubernamentalidad, la cual comprende como "la manera de conducir la conducta de los hombres", no es más que la propuesta de una grilla de análisis para las relaciones de poder en general. ${ }^{4}$

Distintos autores reconocen que, como perspectiva para analizar el poder, la gubernamentalidad se vincula o apoya en dos instancias u objetos de estudio: racionalidades políticas y tecnologías de gobierno (Rose y Miller, 1992; De Marinis, 1999; O'Malley, 
De Prácticas y discursos

Universidad Nacional del Nordeste

Centro de Estudios Sociales

Año 8, Número 11, 2019, Marzo

ISSN 2250-6942
${ }^{5}$ Tomando como inspiración lo elaborado por Tamar Pitch (2003), la noción de cuestión criminal alude a un conjunto de problemas móviles que está integrado por discursos y prácticas, por actores estatales y no estatales, cuyas fronteras son flexibles y porosas. Aunque estas últimas no se encuentran del todo claras, existen términos que delimitan dicho conjunto: delito, control social o control del delito.
2006; Dean, 2010; Sozzo, 2008, 2015), sobre las cuales volveré luego en el siguiente apartado.

Si bien las elaboraciones conceptuales de Foucault en torno a la gubernamentalidad que fueron presentadas hasta aqui son distintas, pudiéndose destacar que una tendria un alcance más restringido (vinculada a la regulación/gestión de la población) y otra, más amplia (entendida como conducción de conductas en general), de todos modos no considero que sean excluyentes entre sí, puesto que ambas están unidas por la inquietud acerca de cómo se ejerce el poder. De hecho, volviendo sobre Valverde (2017: 89-90, traducción propia), ella vincula elocuentemente tales acepciones: "la gubernamentalidad utiliza masas de información sobre las poblaciones -datos agregados- para conducir la conducta de individuos y grupos en gran parte de manera impersonal y para conducir su propio trabajo de política".

Junto a esta noción, otro de los pilares de este artículo lo constituye la criminología. Por ella entiendo tanto un espacio polivalente de saberes referidos a la cuestión criminal ${ }^{5}$ (Marí, 1984) como "una ciencia política [que] tiene como centro de referencia la actividad de gobernar, de gestionar individuos y poblaciones" (Sozzo, 2006: 354). En la misma dirección, y a la vez como inspiración de esta caracterización, Massimo Pavarini (2006) afirma que el discurso sobre la cuestión criminal siempre se tradujo en política criminal, y toda criminología, en práctica criminológica perteneciente al campo de las respuestas a las demandas de orden social y de protección contra la criminalidad.

Partiendo de las mencionadas delimitaciones conceptuales, en este artículo propongo describir dos derivas criminológicas de la gubernamentalidad. La primera de ellas relacionada a la georreferenciación del delito (más comúnmente conocida como la construcción de mapas del delito) y la segunda, referida a la aplicación de una grilla de inteligibilidad economicista sobre los comportamientos delictivos y a la definición del delincuente como homo economicus. Ambas fueron reconstruidas a partir de dos publicaciones editadas desde Argentina, y con eje en el ámbito de la ciudad de Buenos Aires: el Segundo Informe de Conflictividad (2010), producido por el Ministerio Público Fiscal de la Ciudad de Buenos Aires (institución perteneciente al Poder Judicial), y algunos artículos pertenecientes a la Revista de Policía y Criminalística, editada entre 1995 y 2011 por la propia editorial de la Policia Federal Argentina. 
De Prácticas y discursos

Universidad Nacional del Nordeste

Centro de Estudios Sociales

Año 8, Número 11, 2019, Marzo

ISSN 2250-6942
Derivas criminológicas de la gubernamentalidad:

Mapas del delito y grilla de inteligibilidad economicista

Criminological drifts of governmentality: Crime maps and grid of economic intelligibility

\footnotetext{
${ }^{6}$ Encontramos ecos de este proyecto en el primer volumen de Historia de la gubernamentalidad (CastroGómez, 2010).
}

Parafraseando a Jonathan Simon (2011), la posibilidad de trabajar estas publicaciones, a la vez que presentarlas conjuntamente en un mismo artículo, responde al lugar que tienen en una problemática teórica en particular: ambas pueden constituirse como observables de racionalidades y tecnologías tendientes a "gobernar a través de la prevención del delito".

Lejos de aplicar algunas herramientas conceptuales en un dominio empírico para corroborar un determino estado de situación, mi propósito es el de realizar una exploratoria incursión -inspirada y guiada por las elaboraciones conceptuales de Foucault y de autores posteriores- acerca de discursos y prácticas, presentes en las publicaciones consultadas, mediante los cuales se piensa y se pretende gobernar el ámbito de la cuestión criminal.

\section{GUBERNAMENTALIDAD. RACIONALIDADES POLITICAS Y TECNOLOGÍAS DE GOBIERNO}

Enmarcado en el curso Seguridad, territorio, población, el término de gubernamentalidad es introducido por primera vez en la presentación que Foucault hizo de la literatura antimaquiaveliana. Más particularmente, en torno a la emergencia de un arte de gobierno que, desbloqueado a partir del problema de la población y del surgimiento de nuevos saberes, se distinguía del mero ejercicio del poder soberano sobre un territorio delimitado.

Tal centralidad de la población -así como la idea de gobierno como gobierno de la población- le permite conectar al pensador francés estos temas con aquellos que había indagado previamente en el Collège de France, como ser las características de la soberanía y el desarrollo de las disciplinas. Incluso, este aspecto lo lleva a replantear el objetivo del curso del año lectivo 1977-1978: hacer una historia de la gubernamentalidad ${ }^{6}$ (o de la gubernamentalización del Estado), considerando especialmente entre sus etapas al poder pastoral y al arte de gobernar según la razón de Estado, el cual a su vez se apoya en dos dispositivos que lo caracterizan: el sistema diplomático-militar y la ciencia de policía.

Por su parte, en Nacimiento de la biopolítica, Foucault puntualiza al liberalismo como una nueva racionalidad en el arte de gobernar, la cual está ligada a la exigencia de una autolimitación interna de la razón gubernamental. E incursiona, a la vez, en las revisiones acaecidas desde la segunda posguerra mundial, dando cuenta de los proyectos de refundación de la matriz liberal en los contextos 
De Prácticas y discursos

Universidad Nacional del Nordeste

Centro de Estudios Sociales

Año 8, Número 11, 2019, Marzo

ISSN 2250-6942
${ }^{7}$ Un dato para nada menor es que Gordon (1991) señala el uso casi intercambiable que hace Foucault de los términos arte y racionalidad de gobierno, cuyas definiciones explicita. Las artes de gobierno son descriptas como modos de conocer en qué consiste la actividad de gobernar y cómo debiera ser llevada a cabo. La racionalidad de gobierno (o gubernamental), por su parte, es definida como una manera o sistema de reflexionar acerca de la naturaleza de la práctica de gobierno (quién puede gobernar, qué es gobernar, qué o quién es gobernado), capaz de hacer de alguna forma a esa actividad pensable y practicable, tanto para sus practicantes como para aquellos sobre quienes fue practicada. de Alemania, y en menor medida, Francia y Estados Unidos.

En la transición desde un curso al otro -como ya se ha adelantado en la Introducción-, la noción de gubernamentalidad pasa de ser un concepto acotado a tener un sentido más amplio y general, concebido como grilla para analizar las relaciones de poder.

En la síntesis que Colin Gordon (1991: 2, traducción propia) presenta de los antedichos cursos, refiere del siguiente modo a tal definición amplia: "una forma de actividad que se dirige a moldear, direccionar y afectar la conducta de alguna persona o personas". Si bien el autor puntualiza que dicha actividad involucra distintas formas y sentidos de gobierno, destaca que en aquellas clases dedicadas a la gubernamentalidad (o a la racionalidad gubernamental), Foucault centró su atención en el gobierno del dominio político.

Más específicamente aún, señala que el pensador francés aplicó esta perspectiva de análisis sobre distintos dominios históricos: (a) La idea de gobierno como una forma de poder pastoral (en la filosofía griega y en el cristianismo antiguo y primitivo); (b) Razón de Estado y ciencia de policia (en el contexto de la Modernidad temprana europea); (c) Los inicios del liberalismo, considerado como una concepción del arte de gobierno (siglo XVIII) y finalmente, (d) formas de pensamiento neoliberal de posguerra, concebidas como maneras de repensar la racionalidad de gobierno ${ }^{7}$. Para él, lo que une entre sí a estas distintas y discontinuas incursiones es un punto de interés común, encapsulado en la fórmula de "omnes et singulatum", el gobierno de todos y cada uno, en su doble faceta, totalizador/individualizador:

[En] la racionalidad gubernamental moderna [...] se trata simultáneamente de individualizar y totalizar, esto es, de encontrar respuestas acerca de la cuestión de qué es ser gobernado o gobernable para un individuo, para una sociedad o para una población de individuos. (Gordon, 1991: 36, traducción propia)

Del conjunto de interrogaciones sobre estos desarrollos de Foucault, la pregunta acerca de por qué efectuó un desplazamiento conceptual del poder a la gubernamentalidad ha sido ensayada por distintos autores. Así, continuando con lo expuesto por Gordon (1991), el tópico de la gubernamentalidad es introducido como una respuesta a la objeción de que su perspectiva del poder no permitiría dar cuenta de cuestiones globales de la política, y principalmente, las relaciones entre sociedad y Estado. Nikolas Rose y Peter Miller (1992) presentan un punto de vista similar, 
De Prácticas y discursos

Universidad Nacional del Nordeste

Centro de Estudios Sociales

Año 8, Número 11, 2019, Marzo

ISSN 2250-6942
Derivas criminológicas de la gubernamentalidad:

Mapas del delito y grilla de inteligibilidad economicista

Criminological drifts of governmentality: Crime maps and grid of economic intelligibility
${ }^{8}$ Para mayor detalle del mismo, tanto en términos de los enfoques, problemas como limitaciones, remito a los trabajos de Garland (1997), De Marinis (1999), Castro-Gómez (2010) y Rose, O’Malley y Valverde (2012). al señalar que con sus reflexiones sobre la gubernamentalidad, Foucault bocetó una analítica alternativa como respuesta a la necesidad de ampliar la noción de poder, con miras a comprender su ejercicio en las sociedades modernas. Para Pablo De Marinis (1999), un conjunto de problemas teóricos, metodológicos y sobre todo, sociopolíticos -relacionados a la crisis del Estado de Bienestar y a la activación/reactivación de racionalidades politicas y tecnologias de gobierno neoliberales- condujeron al pensador francés a reelaborar su concepto de poder. Senellart (2011) comprende que dicho desplazamiento se produce para dar lugar a un nuevo objeto de estudio, el Estado que, siguiendo lo expuesto por el autor, no tenía cabida en el análisis previo que Foucault hiciera de las disciplinas.

Más allá de cuales sean las razones reseñadas, lo cierto es que tales desarrollos en torno a las elaboraciones conceptuales de la gubernamentalidad -presentes en Seguridad, territorio, población y Nacimiento de la biopolítica- dieron lugar al denominado "efecto Foucault" y a la emergencia de un campo de investigaciones específico: los governmentality studies o también denominada como governmentality literature ${ }^{8}$.

Ya sea que se trate de autores que se posicionan al interior de dicho campo, o aquellos que -provenientes de otros ámbitos académicos- utilizan algunas de sus distintas herramientas teóricometodológicas, reconocen un punto nodal en el hecho de que las problemáticas de la gubernamentalidad puedan ser analizadas en términos de racionalidades políticas y tecnologías de gobierno.

Siguiendo los desarrollos de Pat O'Malley (2006) y Máximo Sozzo $(2008,2015)$, comprendo por las primeras, el conjunto de procesos de pensamiento (concepciones, objetivos, categorías, subjetividades, etc.) desplegados por aquellos que pretenden gobernar. Las tecnologias, por su parte, son formas de hacer dirigidas a intervenir en el mundo físico y social, de acuerdo a rutinas determinadas y que tienen cierta regularidad. En conjunto, se trata de modos de pensar y actuar en torno al problema de gobierno. Tomando como inspiración lo expuesto por David Garland (1997), el campo del control del delito -como parte de los discursos más amplios sobre la cuestión criminal- constituye uno de los dominios posibles para efectuar análisis en términos de las dimensiones de la gubernamentalidad. Más particularmente, al indagar en racionalidades y en tecnologías tendientes a "gobernar a través de la prevención del delito". 
De Prácticas y discursos

Universidad Nacional del Nordeste

Centro de Estudios Sociales

Año 8, Número 11, 2019, Marzo

ISSN 2250-6942
${ }^{9}$ Para un mayor detalle del mismo, se puede acceder online a su contenido mediante la web: https://www. fiscalias.gob.ar/wp-content/uploads/prev/segundoinformeconflictividad.pdf

\section{LA GEORREFERENCIACION DEL DELITO COMO TECNOLOGÍA DE GOBIERNO}

Al tener como punto de partida la elaboración conceptual de gubernamentalidad, tanto en su sentido amplio pero especialmente en cuanto a que se basa en la regulación/gestión de la población y de sus riesgos (Valverde, 2017), la primera deriva criminológica que aquí presento apunta a la georreferenciación del delito (o más comúnmente conocida como la construcción de mapas de delito), la cual concibo como una tecnología mediante la cual se pretende gobernar el ámbito de la cuestión criminal, y más particularmente - para decirlo a partir del título de un libro de Dario Melossi-, una condición para "controlar el delito, controlar la sociedad".

Las características de la misma fueron reconstruidas a partir de la lectura del Segundo Informe de Conflictividad9 ${ }^{9}$ 2010), publicación producida por el Ministerio Público Fiscal de la Ciudad Autónoma de Buenos Aires, institución perteneciente al Poder Judicial.

Una de las ideas que alli aparece como fundamental es aquella que da cuenta que vivimos en un ámbito en el que cada vez hay mayor conflictividad; aludiendo a la información de que en 2009 se recibieron 57921 casos de delitos, contravenciones y faltas, elevándose en un 75\% en solo dos años (2007-2008). Se resalta el hecho de que una de las formas de reducir el conflicto (ya sea que se trate de delitos, contravenciones o faltas) y fortalecer la prevención es estudiando cada lugar, barrio, comuna en específico. En los propios términos en los cuales está expresado en dicha publicación, prevenir es anticiparse a nuevas manifestaciones de la inseguridad.

De esta manera, una de las "recetas" que se plantean para lograr tal prevención del conflicto -y de acuerdo a mis intereses en este artículo, más particularmente el delito- es apelando a la construcción de mapas del delito. En el Segundo Informe de Conflictividad se detallan los procedimientos seguidos para su elaboración:

- Se seleccionaron y utilizaron aquellos casos que tenían registrada correctamente una dirección postal.

- Luego se procedió a la geocodificación, proceso definido como la conversión de direcciones postales en coordenadas geográficas (a partir de lo cual los registros se visualizan en un mapa).

- Para el armado y diseño de los mapas se utilizaron distintos programas informáticos: ArcGIS ArcEditor 9.1 y CrimeStat III, versión 3.1. 
De Prácticas y discursos

Universidad Nacional del Nordeste

Centro de Estudios Sociales

Año 8, Número 11, 2019, Marzo

ISSN 2250-6942
Derivas criminológicas de la gubernamentalidad:

Mapas del delito y grilla de inteligibilidad economicista

Criminological drifts of governmentality: Crime maps and grid of economic intelligibility
${ }^{10}$ En otra publicación elaborada por el Ministerio Público Fiscal (el Segundo Informe Anual de Gestión, 2008-2009) se destaca el mayor aprovechamiento de las herramientas tecnológicas, ilustrado a partir de que le dedican integramente un apartado al "Departamento de tecnologías de la información y las comunicaciones”. Para más detalle remito igualmente a su lectura online: https://es.calameo.com/read/0015792412cdb1991a0e9
- Finalmente, en el análisis estadístico y el modelado espacial se utilizó el método Kernel, que permite representar la densidad de una variable determinada teniendo en cuenta las distancias relativas de los elementos analizados espacialmente.

Estos procedimientos podrian ser leídos en clave del aspecto técnico del gobierno (Dean, 2010), tanto en el sentido de que esos medios técnicos (especialmente informáticos ${ }^{10}$ ) constituyen una condición para gobernar el ámbito de la cuestión criminal -en este caso específico, a través de la prevención del delito- como a partir de la presentación de los límites acerca de lo que es posible hacerse.

En paralelo, Rose y Miller (1992) y De Marinis (1999) aportan otros elementos que permitirían caracterizar la construcción de los mapas del delito como una tecnología de gobierno. Como apertura de telón, se podría señalar que entre los ejemplos de estrategias, mecanismos y procedimientos heterogéneos e ilimitados que buscan hacer operables los programas, Rose y Miller mencionan técnicas de cómputo y cálculo y la invención de dispositivos tales como informes y formas de presentar la información; si bien hacen referencia al respecto a tablas, podriamos destacar la instancia en que el énfasis está puesto en lo visual, como en el caso de los susodichos mapas.

Con mayor detalle, señalan otros aspectos igualmente relevantes al hacer referencia a la inscripción y al cálculo como tecnologías gubernamentales. Por un lado, afirman que "la representación de aquello que va a ser gobernado es un proceso técnico, activo" (Rose y Miller, 1992: 185, traducción propia) que transforma eventos y fenómenos en información; un modo de actuar sobre la realidad, haciendo al dominio en cuestión -en el caso de la presente descripción, el delito- susceptible de ser evaluado, calculado y, en última instancia, intervenido. Por el otro, reconocen que "las figuras transforman el dominio en el cual es aplicado el gobierno" y "son ellas mismas mecanismos que habilitan el establecimiento de relaciones entre distintos fenómenos" (Rose y Miller, 1992: 186, traducción propia), permitiendo así que el delito pueda ser representado como una entidad calculable, y a la vez, puesto en correlación con otros aspectos del espacio urbano metropolitano.

En una similar línea de indagación, De Marinis (1999) señala que la noción de tecnología resulta útil porque permite incluir en el análisis la participación de agentes no humanos, tales como 
De Prácticas y discursos

Universidad Nacional del Nordeste

Centro de Estudios Sociales

Año 8, Número 11, 2019, Marzo

ISSN 2250-6942

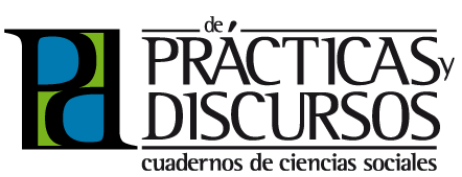

artefactos técnicos y aparatos de registro e inscripción. Destaca igualmente que en las tecnologias de gobierno interviene siempre un elemento de cálculo y previsión. Apoyándose, a su vez, en lo elaborado por Mitchell Dean, puntualiza que el concepto de tecnología de gobierno plantea la relación entre formas de saber y ciertas actividades prácticas y técnicas. Al respecto, es posible destacar que, en la construcción de los mapas del delito, la estadistica y algunas de sus derivaciones cumplieron un rol central; a tal punto de que podemos presentarla como uno de los saberes caracteristicos sobre los cuales se apoya dicha tecnología. A partir de ella, se asignaron números y códigos a distintas zonas de la Ciudad Autónoma de Buenos Aires, con miras a hacer cuantificables o medibles las distintas manifestaciones de la conflictividad (delitos, faltas y contravenciones). Incluso, observamos modelos como el de curva normal y distribuciones de frecuencias: en el Segundo Informe Anual de Gestión 2008-2009, se resalta el hecho de que la construcción de mapas del conflicto no solo permitiria focalizar las zonas más vulnerables e impactadas, sino además determinar el tipo de conflicto más frecuente en ellas.

Podemos señalar así una coincidencia estratégica entre una modalidad de ejercicio de poder en particular (cuyo blanco principal es la gestión/regulación de una población), y una tecnología de gobierno específica (la georreferenciación del delito), en el sentido de que ambas se apoyan en la utilización de los datos agregados de una población y están orientadas al futuro; pues, si los mapas se orientan a la prevención del delito, es porque permitirían anticiparse a nuevas manifestaciones del mismo. Elocuentemente al respecto, en el Segundo Informe de Conflictividad se enuncia que no solo se trata de evitar otros hechos similares, sino también de intervenir a tiempo en contextos problemáticos para que no se desencadenen consecuencias más graves.

En un influyente artículo para quienes buscamos incursionar en la intersección entre gubernamentalidad y criminología, Garland (1997) señala los principales tópicos (o los más relevantes) para quienes investigan el campo del control del delito y de la política penal. Uno de ellos apunta al entrecruzamiento entre estadisticas y biopoder. Apoyándose en lo elaborado por lan Hacking, uno de los aspectos centrales es la noción del conocimiento estadístico como tecnología de gobierno del Estado moderno, en el sentido que formaría la base para problematizaciones políticas. A partir de los 
procesos estadísticos, los fenómenos colectivos de la población son reagrupados y clasificados; en el caso en particular de los mapas, mediante la distinción de zonas calientes y zonas frías, ilustrando un recuento y ordenación acerca de donde se cometen mayores y menores cantidades de delitos. Otro de los tópicos que desarrolla Garland es el de las tecnologías de seguros y de los razonamientos basados en el riesgo. De ellos me interesa detenerme en la noción de riesgo, puesto a que tiene un cierto protagonismo en la construcción de los susodichos mapas. De hecho, forma parte de los objetivos a partir de los cuales son presentados: ubicar espacialmente los focos de conflicto, conocer de un modo adecuado los riesgos de victimización y organizar con mayor eficiencia la actividad policial.

Junto al modelado técnico e informático del espacio urbano y metropolitano, y el ensamble de la tecnología con otros actores institucionales (entre los cuales se hace mención explícita a la policía), los mapas del delito permitirán dar cuenta de los riesgos de que en determinadas zonas una/s persona/s se conviertan en víctimas del delito. Pudiendo ser pensado así como un observable empírico de la actividad gubernamental tematizada a la manera de gestión de riesgos: los análisis de cuáles son los factores de riesgo y cuáles los grupos que mayormente lo producen, hacen posible, siguiendo a Garland (1997: 182, traducción propia), "gobernar la salud y la criminalidad en el nivel de la población".

Si, por otro lado, nos apoyamos en lo elaborado por Pat O'Malley (2006: 21), el riesgo en sí mismo puede ser conceptualizado como una "modalidad de gobierno de los problemas a través de la predicción y la prevención". El autor reconoce que, como tecnología de gobierno, el riesgo fue central en el desarrollo del marco analítico de la gubernamentalidad. Menciona al respecto distintos capitulos incluidos en The Foucault Effect. Studies on Governmentality, los cuales se orientaban al riesgo desde la perspectiva de los seguros (Defert y Ewald) y la psiquiatría (Castel). Particularmente, el trabajo del propio 0'Malley estuvo orientado a los entrecruzamientos entre el riesgo y el área de gobierno de la justicia penal. Por mi parte, puedo sostener que la lectura del Segundo Informe de Conflictividad permitiria indagar en la imbricación entre las tecnologias de construcción de 
De Prácticas y discursos

Universidad Nacional del Nordeste

Centro de Estudios Sociales

Año 8, Número 11, 2019, Marzo

ISSN 2250-6942
${ }^{11}$ Este dominio empírico, así como algunos aspectos de esta segunda deriva criminológica de la gubernamentalidad, forman parte de mi tesis para la obtención del título de Doctor en Ciencias Sociales (Universidad de Buenos Aires), la cual está siendo dirigida por Néstor Cohen y Máximo Sozzo.

Para mayor detalle del contenido de la Revista Policía y Criminalística, remito a García (2012) y Abiuso (2016). mapas del delito y la del riesgo. Encontramos un puntapié inicial de este entrecruzamiento -el cual requiere ulteriores desarrollos- en el siguiente fragmento:

Lo que es especifico del riesgo [...] es que se trata de una técnica estadística y probabilística, a través de la cual grandes cantidades de eventos son clasificados en una distribución, que a su vez es utilizada como un medio para hacer predicciones probabilísticas. (O’Malley, 2006: 31)

Desde otra perspectiva de indagación, la confección de tales mapas es situada al interior de las variadas propuestas de la derecha criminológica para enfrentar al delito, como formando parte de las estrategias neoliberales de control social (Pegoraro, 2001). Este constituye un aspecto interesante porque permitiria, al menos, sugerir que la georreferenciación del delito puede ser analizada tanto como observable de una tecnología mediante la cual se pretende gobernar el ámbito de la cuestión criminal, como un ejemplo de lo que Foucault (2012: 304) denominó -en el marco de sus reflexiones en torno al neoliberalismo penal- una intervención de tipo ambiental: "la tecnología que será menester utilizar no es la disciplina-normalización, es la acción sobre el ambiente. Modificar la manera de repartir las cartas del juego, no la mentalidad de los jugadores".

A partir de la lectura del mismo curso donde sostuvo esta noción, Foucault nos invita a indagar en la otra deriva criminológica de la gubernamentalidad que aquí querría presentar: la aplicación de una grilla de inteligibilidad economicista sobre comportamientos delictivos y la definición del delincuente como un homo economicus.

Si la construcción de los mapas del delito se encontraba vinculada teóricamente a la dimensión de la tecnologías de gobierno, esta segunda deriva apunta al plano de las racionalidades politicas mediante las cuales se piensa la actividad de "gobernar a través de la prevención del delito", y más concretamente, los planes y categorías de alteridades que en ellas se ponen en juego.

PROCESOS DE PENSAMIENTO: OBJETIVOS Y CONCEPCIONES EN TORNO A LA CRIMINALIDAD

Esta segunda deriva fue reconstruida a partir del análisis de la Revista de Policía y Criminalística" ${ }^{11}$, editada entre 1995 y 2011 por la propia editorial de la Policía Federal Argentina. Como ac- 
tividad de gobierno, en tanto impulsora de "técnicas y procedimientos destinados a dirigir la conducta de los hombres" (Foucault, 2014: 359), la institución policial gestiona la población delimitando identidades y alteridades.

En tal sentido, Mariana Galvani (2016) nos invita a describir las clasificaciones acerca del otro que se ponen en juego en la propia configuración de la identidad policial. Estableciendo un puente entre pasado y presente, y enfocándose en las representaciones que constituyen la forma de existir de la institución policial en tres dominios empíricos distintos (entrevistas, normativa legal y medios de comunicación), la autora sostiene que los otros no tienen una única forma de ser definidos o entendidos por los policias. Existen otros deseables -la sociedad a la cual hay que defender-, y otros no deseables, de los cuales hay que defenderla. Orientándose en la descripción de estos últimos, Galvani (2016: 112) afirma que "nos interesa mostrar tres momentos históricos y rastrear alli esas alteridades indeseadas que reconocemos como centrales de y en la propia historia de la institución": el vago, los lunfardos, los militantes anarquistas, los terroristas y los jóvenes delincuentes.

Tomando este como un antecedente ineludible, mi interés radica en reconstruir algunos de los modos en que, desde la susodicha revista, se tematizaban distintos objetivos para hacerle frente a la criminalidad; y, en la misma dirección, cómo definían a la figura del delincuente. Respecto al primer tópico, me propongo recuperar la perspectiva del -en ese entonces- jefe de la Policía Federal Argentina, Adrián Juan Pelacchi, como una manera de ilustrar otra forma de pensar el gobierno de la cuestión criminal (y más particularmente el aumento del delito) desde una lógica preventiva. En cuanto al segundo, y apoyándome en la lectura de Nacimiento de la biopolítica, querría destacar aquellos artículos que puedan constituir un interesante observable de la aplicación de una grilla de inteligibilidad economicista sobre los comportamientos delictivos.

\section{COMUNIDAD Y PREVENCIÓN EN EL PLAN DE LA POLICÍA FEDERAL ARGENTINA}

En distintas ocasiones, Pelacchi $(1995 a, 1996,1997)$ destaca la importancia y necesidad de un marco más amplio que la mera represión del delito cuando este ocurre; en tal sentido, señala que para reducir la criminalidad y prevenir el delito en los 
De Prácticas y discursos

Universidad Nacional del Nordeste

Centro de Estudios Sociales

Año 8, Número 11, 2019, Marzo

ISSN 2250-6942

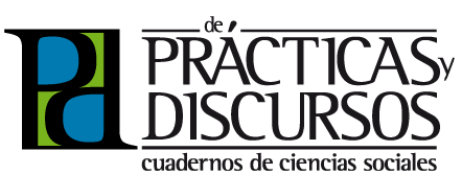

órdenes sociales contemporáneos hay que intervenir sobre las condiciones y factores sociales desfavorables, entre los cuales sobresale resaltar, pues están imbricados en el punto de vista del autor, la marginalidad y las inmigraciones ilegales.

Para efectivizar y llevar adelante esta estrategia de control del delito, se aboga por un modelo de "policía comunitaria". Siguiendo a Sozzo (1999, 2000), este último puede ser definido como todo aquello que mejora las relaciones y la confianza entre la institución policial y la comunidad local. Más particularmente, señala que uno de sus elementos sustanciales -en tanto técnica de intervención- es el desarrollo de relaciones de cooperación entre la Policía y otros actores en la actividad de prevención y en la generación de procedimientos de consulta con la comunidad local, en términos de cuáles serian las prioridades y los principales problemas.

La prevención y la comunidad constituyen así los dos pilares del abordaje de la institución policial que propone Pelacchi (1997: 14), "avanzaremos con mayor enjudia y convicción que nunca en nuestras posibilidades prevencionales y fundamentalmente en la ejecución de políticas comunitarias". La consigna de "gobernar a través de la prevención del delito" se yuxtapone así con "gobernar a través de la comunidad". Sería interesante indagar en el futuro estos aspectos en relación con la reactivación comunitaria a la cual refiere Pablo de Marinis (1999), como formando parte de la recodificación neoliberal del Estado y de la redefinición del lugar del sujeto.

\section{GRILLA DE INTELIGIBILIDAD ECONOMICISTA}

En la clase del 21 de marzo de 1979 de Nacimiento de la biopolítica, Foucault destaca una característica central del neoliberalismo norteamericano: su ambición constante en generalizar la forma económica del mercado. Uno de los rasgos que implica es que esta generalización funciona como principio de inteligibilidad, principio de desciframiento de las relaciones sociales y los comportamientos individuales. En las propias palabras del autor, "una tentativa de desciframiento en términos económicos de comportamientos sociales tradicionalmente no económicos" (Foucault, 2012: 284). 0 como refiere, de manera más sintética, se trataría de un análisis economicista de lo no económico.

Al interior de este escenario conceptual son destacados ejemplos de distintos campos, tales como la relación formativa o relación educacional y el matrimonio. Dados mis intereses en este artí- 
culo, me interesa detenerme en los análisis que algunos autores neoliberales hicieron de la criminalidad y del funcionamiento de la justicia penal, los cuales fueron reseñados y caracterizados por Foucault del siguiente modo.

En primer lugar, son análisis que se definen como un retorno a los reformadores del siglo XVIII, siendo mencionados ilustrativamente las figuras de Cesare Beccaria y Jeremy Bentham. En estos últimos se trataba de un análisis o una reflexión de estilo económico sobre la política o el ejercicio del poder. Criticaban, en nombre de una lógica y una racionalidad económica, el funcionamiento de la justicia penal. En este sentido, advertían datos acerca del costo de la delincuencia, el costo de la propia práctica judicial, así como realizaban una crítica general referida a la poca eficacia del sistema punitivo. Esos reformadores buscaban un sistema penal cuyo costo fuera el más bajo posible; dentro de este esquema la ley sería la solución más económica para que el castigo sea eficaz. La ley, en tanto principio económico, es un elemento que lleva a Foucault a afirmar que para estos pensadores el homo penalis sería un homo economicus.

En relación con este último aspecto, resulta central incorporar uno de los argumentos que presenta Pasquale Pasquino (1991) acerca de la historia del nacimiento de la criminología. En ella, efectúa una distinción entre las figuras del homo penalis y homo criminalis. Con eje en la teoría clásica de Derecho penal, la primera se encuentra emparentada al ciudadano y al establecimiento del contrato social: el homo penalis existe potencialmente en cada uno de nosotros, cualquiera puede cometer un delito como resultado de un cálculo erróneo. En ese marco, el delincuente no es pensado como un sujeto diferente a los demás, sino como una elección equivocada de alguien dotado de plena racionalidad. El homo penalis no se presta como objeto de una forma especializada de saber; aquello que sirve para explicar su accionar es una antropología general apoyada en los pilares de libre albedrío, racionalidad y responsabilidad; la misma que permitiría explicar el comportamiento del homo economicus.

En cambio, la figura del homo criminalis se constituye como objeto privilegiado de un saber especializado, la criminología, cuya génesis el autor sitúa hacia fines del siglo XIX. Cuestionados los antedichos pilares, y con eje en la defensa social y 
De Prácticas y discursos

Universidad Nacional del Nordeste

Centro de Estudios Sociales

Año 8, Número 11, 2019, Marzo

ISSN 2250-6942

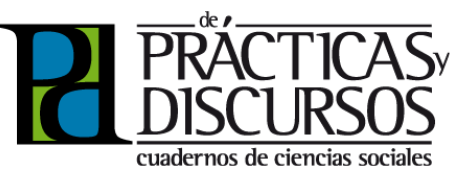

la etiología del delito, el homo criminalis es definido como un individuo diferente respecto del resto de la población: conceptualizado, entre otros modos, como un salvaje o un anormal. Este recorrido histórico que plantea Pasquino resulta interesante para problematizar las maneras de definir al delincuente (y en un plano más amplio, sus vínculos con el gobierno de la cuestión criminal y de la gestión de la población), pudiéndonos preguntar si asistimos actualmente a discursos que evidencian un alejamiento del homo criminalis y de acercamiento, como retorno a los clásicos, al homo penalis. 0 si, más bien, coexisten ambas figuras en un mismo tiempo y espacio. Una discusión demás relevante para transitar la historia de los pensamientos criminológicos.

Continuando el recorrido, y volviendo sobre Foucault, otra característica del análisis de los autores neoliberales consiste en retomar el filtro utilitario que habia sido el de Beccaria y Bentham. Como continuidad, buscan mantener el análisis del problema del crimen dentro de una problemática económica. ¿A partir de cuáles mecanismos o siguiendo cuáles direcciones? A partir de una definición de crimen (como ejemplo, podemos citar aquella que propone Gary Becker y que Foucault recupera): toda acción que hace correr el riesgo a un individuo de ser condenado a una pena. La perspectiva a la cual se adscriben los análisis neoliberales en materia penal es aquella que se sitúa en quien comete el crimen o va a cometerlo; pero no en términos de aquellos factores (biológicos, sociológicos, psicológicos, etc.) que lo llevarian al delito, sino que el criminal es cualquier persona que invierte en una acción, esperando de ella una ganancia y aceptando el riesgo de una pérdida. Solo se toma el comportamiento del individuo en tanto comportamiento económico. Dado el lugar central que ocupa asi lo económico, Foucault (2012: 292) sostiene que "si el individuo va a ser gubernamentalizable, si se va a poder tener influjo sobre él, será en la medida y sólo en la medida en que es homo economicus".

Para el pensador francés, este conjunto de mutaciones trae aparejadas una serie de consecuencias, entre ellas, la borradura antropológica del criminal, ya no importara indagar quién es, no se trata de definirlo en su carácter de individualidad, sino que únicamente se toma su accionar, y este último a la vez, como accionar exclusivamente económico, es decir, como homo economicus que prefiere y renuncia a la vez. 


\section{MARGINALIDAD Y DEFINICION DEL DELINCUENTE COMO HOMO ECONOMICUS}

En estos últimos apartados voy a detallar algunos posibles observables de la aplicación de esta grilla, tomando para ello distintos artículos de la Revista de Policía y Criminalística.

Por un lado, podría destacarse la caracterización que Pelacchi (1995b) presenta de la marginalidad, entendida como una condición socio-económica que predispondría al delito: "Sumamos un creciente sector social proveniente del interior y de paises limitrofes que se encuentra aislado y en condiciones económicas de marginalidad que lo predisponen al delito".

En otro artículo suyo (Pelacchi, 1997), el autor tematiza la marginalidad aludiendo a las condiciones precarias de residencia en la ciudad de Buenos Aires. De esa manera, se señala que conglomerados tales como villas de emergencia, barrios carenciados, asentamientos y complejos habitacionales se encuentran habitados por personas procedentes del interior del pais y ciudadanos "indocumentados" provenientes en su mayoría de países vecinos. En el mismo sentido, se destaca que de las 8000 personas que usurparon viviendas en la Capital Federal, entre el 65\% y 70\% son extranjeros "ilegales". Para él, "de la marginalidad instalada en un sistema social, es muy dificil esperar o construir algo positivo" (1997: 7). Especialmente en términos del impacto que la usurpación tendría en materia de seguridad.

Orientándonos en esta primera dirección, la grilla de inteligibilidad economicista es aplicada de manera sutil al comportamiento delictivo, el cual terminaría siendo explicado por el accionar de variables casi exclusivamente económicas.

Por otro lado, resulta significativo puntualizar aquellos artículos donde el delincuente es definido más íntegramente como un homo economicus; los cuales podríamos destacar como formando parte de discursos y lenguajes criminológicos analizados por David Garland (2012): las criminologías de la vida cotidiana o la criminología del sí mismo.

En uno de ellos, Francisco Thoumi (2002) propone una aproximación a las ventajas competitivas de las drogas ilegales en los países andinos (Perú y Bolivia), al interior de la cual presenta algunos de los lineamientos acerca de la utilización de un modelo económico explicativo. Al efectuar este recorrido, señala distintas nociones: evaluación, beneficios, costos, riesgos. 
De Prácticas y discursos

Universidad Nacional del Nordeste

Centro de Estudios Sociales

Año 8, Número 11, 2019, Marzo

ISSN 2250-6942

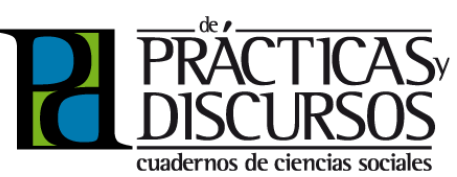

Como sintesis de distintos argumentos que presenta a lo largo del artículo, Thoumi (2002: 45) resalta lo siguiente:

La aproximación a la actividad económica ilegal está basada en la premisa de que muchas acciones criminales están motivadas por las ganancias económicas esperadas y en que estos crímenes pueden ser analizados en formas similares a otras actividades económicas. Desde este punto de vista, las decisiones para cometer un crimen se basan en una evaluación de los beneficios esperados de los crímenes, comparada con los costos esperados y los riesgos involucrados.

En este punto, el criminal es concebido como un homo economicus que realiza una inversión entre los costos y las ganancias, así como una evaluación entre los beneficios y los riesgos.

Nuevamente, acerca de la forma de concebir al delincuente, Bordas Martínez (2007) reconoce algunos lineamientos de la aplicación de la teoría de la elección racional. En tal sentido, afirma que los delincuentes, al igual que el resto de la población, persiguen su propio beneficio y cuando toman decisiones pretenden que sean el resultado de haber sopesado racional y anteriormente riesgos y compensaciones. Existe para el autor un cálculo entre los riesgos y los estímulos que suponen la comisión de delitos. A la vez, subraya el desplazamiento -presente en los análisis comentados por Foucault- del foco del delincuente al delito.

En conjunto, analizar los discursos presentes en los medios institucionales de comunicación de la Policia Federal Argentina puede resultar un interesante camino para indagar en las concepciones que se producen en torno a la figura del delincuente, y a la vez, los objetivos y propósitos con los cuales se piensa gobernar el ámbito de la cuestión criminal, por ejemplo promoviendo un acercamiento de la institución a la comunidad.

\section{REFLEXIONES FINALES}

Al tener presente como punto de partida las elaboraciones conceptuales de Michel Foucault en torno a la gubernamentalidad, entendiendo por ella tanto una modalidad de ejercicio de poder político -cuyo blanco principal es la población- como una manera de conducir las conductas en general, propuse en este artículo una incursión exploratoria hacia dos de sus derivas criminológicas: la georreferenciación del delito y la aplicación de una grilla de inteligibilidad economicista sobre los comportamientos delictivos. 
Ambas fueron reconstruidas mediante la lectura de dos publicaciones: el Segundo Informe de Conflictividad y algunos artículos pertenecientes a la Revista de Policia y Criminalistica. Tomando en consideración que la analítica de la gubernamentalidad se interesa en las superficies, "en las palabras usadas para describir los problemas, los discursos de gobierno en términos de los cuales los sujetos son caracterizados, las categorías que son usadas para explicar las políticas" (O'Malley, 2006: 30), este artículo fue una primera aproximación al análisis de racionalidades políticas y tecnologías de gobierno, discursos y prácticas a partir de las cuales se piensa y se pretende gobernar, más que el delito, el conjunto más amplio de la cuestión criminal.

Querría concluir -aunque sea de manera momentánea- invitando a reflexionar acerca de los procesos de traducción de determinadas elaboraciones conceptuales (principalmente, en torno a las maneras de comprender gubernamentalidad) que son aplicadas en un contexto distinto al cual fueron originariamente desarrolladas. A mi juicio, únicamente el momento con el encuentro empírico permite dar cuenta del alcance, de las potencialidades y limitaciones, de nociones que atraviesan viajes culturales de una circunscripción geográfica a otra.

BIBLIOGRAFÍA

ABIUSO, F.L. (2016). Un modo de construcción de la otredad. Representaciones de la Policía Federal Argentina acerca de los nexos entre la inmigración sudamericana y la delincuencia. Delito y Sociedad, (25) 42, 103-129.

BORDAS MARTÍNEZ, J. (2007). La personalidad del terrorista en la sociedad calidoscópica actual. Revista de Policía y Criminalística, (11) 18, 12-19.

CASTRO-GÓMEZ, S. (2010). Historia de la gubernamentalidad. Razón de Estado, liberalismo y neoliberalismo en Michel Foucault. Bogotá: Siglo del Hombre Editores.

DEAN, M. (2010). Governmentality. Power and Rule in Modern Society. Londres: Sage.

DE GIORGI, A. (2005). Tolerancia cero. Estrategias y prácticas de la sociedad de control. Barcelona: Virus editorial. 
DE GIORGI, A. (2006). El gobierno de la excedencia: postfordismo y control de la multitud. Madrid: Traficantes de Sueños.

DE MARINIS, PABLO (1999). Gobierno, gubernamentalidad, Foucault y los anglofoucaultianos (Un ensayo sobre la racionalidad politica del neoliberalismo). En García Selgas, F. y Ramos Torre, R. (eds.) Globalización, riesgo, rejtexividad. Tres temas de la teoría social contemporánea (pp. 73-103). Madrid: Centro de Investigaciones Sociológicas.

FOUCAULT, M. (2010). Defender la sociedad. Curso en el Collège de France 1975-1976. Buenos Aires: Fondo de Cultura Económica.

(201 1a). Seguridad, territorio, población. Curso en el Collège de France 1977-1978. Buenos Aires: Fondo de Cultura Económica.

(2011b). Historia de la sexualidad 1. La voluntad de saber. Buenos Aires: Siglo XXI.

(2012). Nacimiento de la biopolítica. Curso en el Collège de France 1978-1979. Buenos Aires: Fondo de Cultura Económica.

(2014). Del gobierno de los vivos. Curso en el Collège de France 1979-1980. Buenos Aires: Fondo de Cultura Económica.

GALVANI, M. (2016). Cómo se construye un policía. La Federal desde adentro. Buenos Aires: Siglo XXI.

GARCíA, B. (2012). Criminalización de la inmigración. La construcción de la figura del inmigrante desde la perspectiva de la Policía Federal Argentina. Derecho y barbarie, 4, 20-27.

GARLAND, D. (1997). Governmentality and the Problem of Crime. Theoretical Criminology, (1) 2, 173-214.

GARLAND, D. (2012). La cultura del control. Crimen y orden social en la sociedad contemporánea. Barcelona: Gedisa.

GORDON, C. (1991). Governmental Rationality: an Introduction. En Graham Burchell, C.G. y Miller, P. (eds.) The Foucault Effect: Studies in Governmentality (pp. 1-51). Chicago: University of Chicago Press. 
MARÍ, E. (1984). Michel Foucault: El espacio polivalente de la criminología (nota necrológica). Doctrina Penal: teoría y práctica en las ciencias penales, 7, 447-451.

MINISTERIO PÚBLICO FISCAL (2009). Segundo informe anual de gestión (2008-2009). Buenos Aires: Autor.

(2010). Segundo informe de conflictividad. Buenos Aires: Autor.

O'MALLEY, P. (2006). Riesgo, neoliberalismo y justicia penal. Buenos Aires: Ad-Hoc.

PAVARINI, M. (2006). ¿Vale la pena salvar a la criminología? En Sozzo, M. (coord.) Reconstruyendo las Criminologías Críticas (pp. 15-42). Buenos Aires: Ad-Hoc.

PASQUINO, P. (1991). Criminology: The Birth of a Special Knowledge. En Graham Burchell, C.G. y Miller, P. (eds.) The Foucault Effect. Studies in governmentality (pp. 235-251). Chicago: The University of Chicago Press.

PEGORARO, J. (2001). Derecha criminológica, neoliberalismo y política penal. Delito y Sociedad, (10) 15/16, 141-160.

PELACCHI, A. (1995A). La seguridad en Buenos Aires. Revista de Policia y Criminalistica, 1, 58-66.

(1995b). Plan estratégico para la Policía Federal Argentina 1996/1997. Políticas y cursos de acción dirigidos a la comunidad. Revista de Policia y Criminalística, 2, 4-8.

(1996). Violencia y criminalidad, policía y participación de la comunidad. Revista de Policía y Criminalística, 3, 4-8.

(1997). Violencia y criminalidad. ¿Qué sucederá con nuestras sociedades actuales? Revista de Policía y Criminalística, 4, 4-14.

Pitch, T. (2003). Responsabilidades limitadas. Actores, conflictos y justicia penal. Buenos Aires: Ad-Hoc. 
ROSE, N. Y MILLER, P. (1992). Political Power Beyond the State: Problematics of Government. British Journal of Sociology, (42) 2, 173-205.

ROSE, N.; O'MALLEY, P. Y VALVERDE, M. (2012). Gubernamentalidad. Astrolabio Nueva Época, 8, 113-152.

SENELLART, M. (2011). Situación de los cursos. En Foucault, M. Seguridad, territorio, población. Curso en el Collège de France 19771978 (pp. 417-453). Buenos Aires: Fondo de Cultura Económica.

SIMON, J. (2011). Gobernar a través del delito. México DF: Gedisa.

SOZZO, M. (1999). ¿Hacia la Superación de la Táctica de la Sospecha? Notas sobre Prevención del Delito e Institución Policial. En Detenciones, facultades y prácticas policiales en la ciudad de Buenos Aires (pp. 3-41). Buenos Aires: CELS.

(2000). Seguridad Urbana y Tácticas de Prevención del Delito. Cuadernos de Jurisprudencia y Doctrina Penal, 10, 17-82.

(2006). Tradutore Traditore. Traducción, Importación Cultural e Historia del Presente de la Criminología en América Latina. En Sozzo, M. (coord.) Reconstruyendo las Criminologías Críticas (pp. 353-431). Buenos Aires: Ad-Hoc.

(2008). Inseguridad, prevención y policía. Quito: Flacso Ecuador.

(2015). Locura y crimen: nacimiento de la intersección entre los dispositivos penal y psiquiátrico. Buenos Aires: Didot.

THOUMI, F. (2002). Ventajas competitivas de las drogas ilegales en los paises andinos: el Estado, las instituciones sociales y el capital social. Revista de Policía y Criminalistica, (8)10, 39-60.

VALVERDE, M. (2017). Michel Foucault. Routledge Key Thinkers in Criminology. Nueva York: Routledge. 